\title{
On the role of geometry in geo-localization
}

\author{
Moti Kadosh ${ }^{1}(\bowtie)$, Yael Moses ${ }^{2}$, and Ariel Shamir ${ }^{2}$ \\ (c) The Author(s) 2020.
}

\begin{abstract}
Consider the geo-localization task of finding the pose of a camera in a large 3D scene from a single image. Most existing CNN-based methods use as input textured images. We aim to experimentally explore whether texture and correlation between nearby images are necessary in a CNN-based solution for the geo-localization task. To do so, we consider lean images, textureless projections of a simple 3D model of a city. They only contain information related to the geometry of the scene viewed (edges, faces, and relative depth). The main contributions of this paper are: (i) to demonstrate the ability of CNNs to recover camera pose using lean images; and (ii) to provide insight into the role of geometry in the CNN learning process.
\end{abstract}

Keywords geo-localization; geometry; CNN-based solutions; synthetic lean images

\section{Introduction}

Imagine that you are brought blindfolded to a street corner of a city you know well. Now, you remove the blindfold. Can you tell where you are? This is the geo-localization task. In computer vision, this amounts to estimating the position (and sometimes the orientation too) of a camera, given its current view. Although localization devices such as global positioning systems have improved significantly over recent years, they often do not work well in city scenes and do not provide highly accurate results. Autonomous cars, drones, and IoT devices are expected to benefit tremendously from the ability to determine their poses (position \& orientation)

1 Department of Electrical Engineering, Tel-Aviv University, Tel-Aviv, Israel. E-mail: moti.kadosh84@gmail.com (凹).

2 The Efi Arazi School of Computer Science, The Interdisciplinary Center Herzliya, Herzliya, Israel. E-mail: Y. Moses, yael@idc.ac.il; A. Shamir, arik@idc.ac.il.

Manuscript received: 2020-07-18; accepted: 2020-09-12 accurately in their environments.

Human or machine solution of the geo-localization problem can use appearance cues (e.g., a unique building texture), geometric cues (e.g., a unique building shape), or both. Significant improvements were obtained in object recognition, scene recognition, and localization tasks, largely by exploiting the appearance of the scene rather than just its edges (e.g., using color, texture, and image features such as SIFT [1-3]). Later, these methods were improved by adding coarse geometric constraints to the image features (e.g., Refs. [4, 5]). Nowadays, methods are often based on machine learning, in particular convolutional neural networks (CNNs), where the input is typically an unprocessed textured image. We expect that both appearance and geometry play an important role in these methods.

The main goal of this paper is to study the role of geometry, in contrast to that of texture, in a CNN solution to the geo-localization task (rather than to propose a working system for application purposes). We study an end-to-end deep CNN for geo-localization, ignoring the often available texture of the scene. To do this, we consider only lean images, which mostly contain information related to the geometry of the scene while lacking texture or rich geometric details. In practice, we use a city scene and consider two types of binary images that consist of the edges forming the buildings' outlines, and the buildings' facades. In addition, we also consider depth images that contain more geometric information. Examples of the three types of lean images are shown in Fig. 1. Note that in the first row, the view contains dominant landmarks, while the second row shows very little distinct information that might be expected to assist localization. Such non-distinctive views are very common in large environments such as a city, making localization with lean images very challenging. 


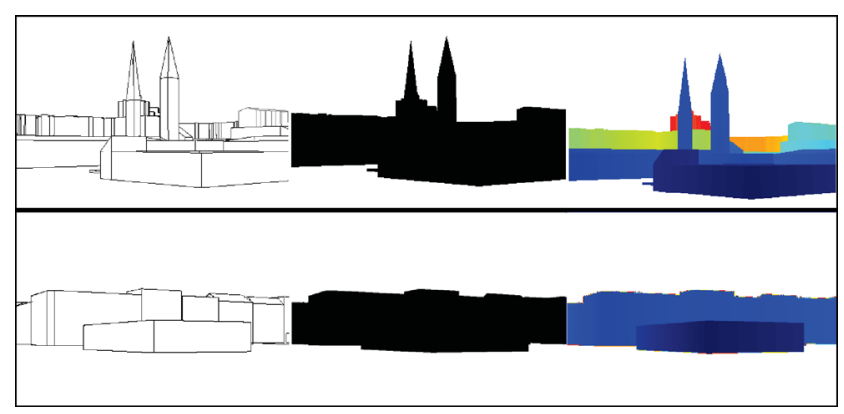

Fig. 1 Lean images contain mostly geometric features: edges (left), faces (center), and depth information (right). We train a CNN to solve the localization problem using such images alone.

Further note that we deliberately do not consider real images or synthetic images with texture, since our goal is to study only the information available from purely geometric data.

Our lean images are obtained simply by projecting an untextured 3D mesh model of Berlin onto various positions in the scene (see Section 3). Using such a model allows us to study the role of geometry for geo-localization in a controlled manner and at a larger scale than ever before, both in terms of the area covered (many city streets) and in terms of the number of images (up to hundreds of thousands). A bird's-eye view of one of the areas is shown in Fig. 2

Neural networks have been shown to be effective in geo-localization tasks (e.g., Refs. [6-9]). A typical geo-localization solution will return the pose of a camera given a novel view (not in the training set). In this paper we ask: (i) can a CNN generalize and support geo-localization in large environments using lean images that contain only geometric and spatial data? (ii) is it likely that geometric information is used by the CNN to solve this task? However, to

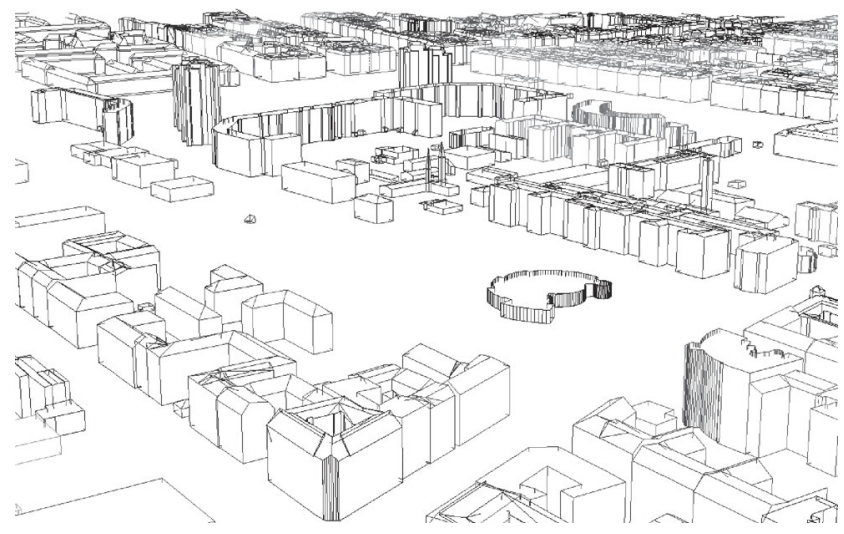

Fig. 2 Projection of the 3D model of one of the areas we used onto a bird's-eye view. better understand the geo-localization task, we also consider the memorization capacity of the network for previously seen images. This can be regarded as image retrieval from a database of all available views of the city. A naive classic solution would store all images and then perform a brute-force search in the database. However, this is inefficient and can become infeasible as the database gets larger. A compact representation and an efficient search method are clearly desired. The question we address in this case is (iii) can CNNs be used to memorize lean images?

As our results show (see Section 6), we found positive answers to all these questions, but the results depend on the number of images and their sampling density. We believe this indicates that networks can learn some sort of a spatial map for an area using only geometric data, since no colors or textures are available in our data. The success of geo-localization also depends on the particular location. Figure 3 shows how certain positions in the streets of a city are more recognizable than others.

Our paper presents an empirical study regarding the information that can be used by CNNs to build an internal representation; we do not propose a practical solution based on lean images. The main contributions of our study are: (i) showing that lean images contain sufficient information for solving memorization and geo-localization tasks,

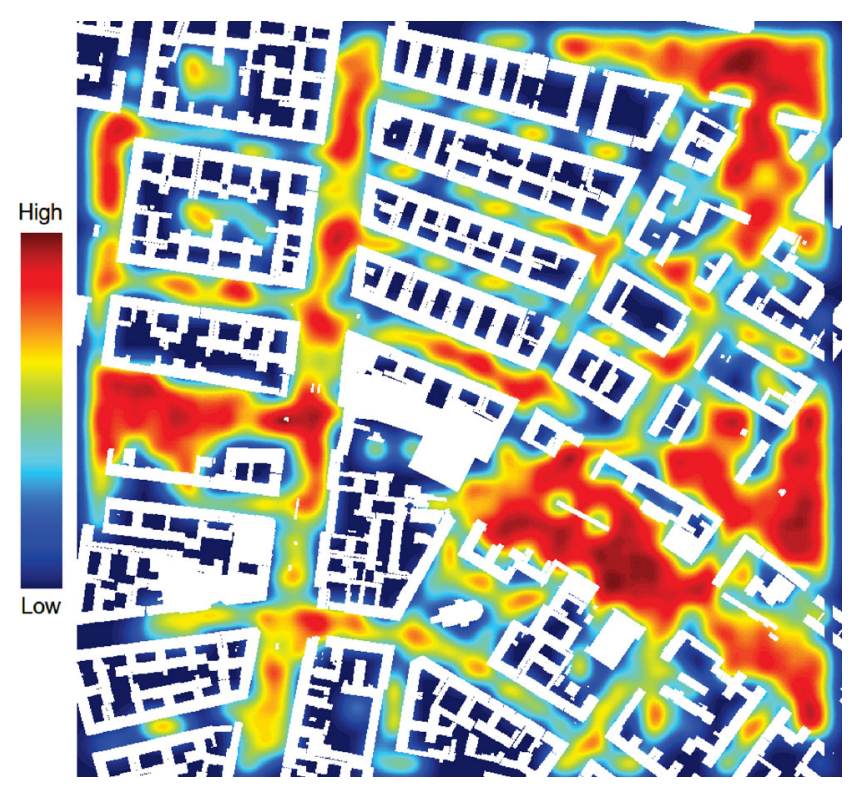

Fig. 3 Top view of a city area, with buildings in white. Color indicates localization success rate of a $\mathrm{CNN}$, from high (red) to low (blue). For instance, note how open spaces are more distinctive than narrow streets. 
(ii) proposing a systematic method to study the role of geometry in CNNs for geo-localization tasks, and (iii) demonstrating the power of CNNs to use geometric information to build internal representations of data.

\section{Related work}

Place (e.g., the Eiffel Tower) recognition can be regarded as a coarse geo-localization task. Finding images of the same place is a basic tool for solving this task. Classic approaches use visual features to represent each image in a set of images of a given location (e.g., by a bag of words) and then match a target image to the stored representations (e.g., Refs. $[1,2,10,11])$. Hayes and Efros [12] were the first to address the place recognition task using millions of geo-tagged images, based on various visual features (e.g., tiny images, color histograms, line features, gist descriptors). Bergamo et al. [13] proposed a new technique for learning a discriminative code-book for local feature descriptors, specifically designed for scalable landmark classification. Dictionary structure varied to achieve faster results. Sivic and Zisserman [10] introduced a text retrieval approach to object matching in videos. Their approach was immediately applied to object detection problems and to scene recognition and localization in datasets. Se et al. [1] formulated a dynamic SIFT [2] database to address the uncertainty in each SIFT landmark due to occlusion and updated it on the fly according to new data. In our study, we consider the geo-localization task where both position and orientation of a camera with respect to a scene should be estimated.

Memorization of image data-sets is often performed using a manually engineered image representation (e.g., a dictionary of image features) and an image retrieval approach, including a metric between the stored representation and a target one (e.g., Refs. [2, 3, 10-12, 14-16]). We show through various experiments that neural networks are able to efficiently create such a representation.

A possible way of solving the geo-localization task, where both position and orientation of a camera with respect to a scene should be estimated, is to use triangulation with images with known pose (e.g., Ref. [14]). In most studies, 3D models of the scene are used by means of point-clouds (e.g., Refs. [17-20]), digital elevation maps (DEM) (e.g.,
Refs. [5, 21]), or full 3D models (e.g., Refs. [4]). One of the main challenges of these works is to develop an efficient method of computing 2D to 3D feature matching. New 3D feature representations have also been developed (e.g., Refs. [17, 18]). Bansal and Daniilidis [5] introduced a feature more closely related to the lean images we consider. It consists of $3 \mathrm{D}$ corners and direction vectors extracted from a DEM, to be matched geometrically to the corners and roofline edges of buildings visible in a street-level query image. Efficiency and robustness become even more important when dealing with a city-scale 3D model. A fast method for inlier detection that enables solution of the correspondence problem on such a scale was suggested by Ref. [22]. A recent survey on existing localization methods can be found in Ref. [23].

One of the key ideas that bypasses the challenge of defining an efficient and robust 2D to 3D feature matching approach required by the abovementioned methods is to use an end-to-end CNN solution that performs both feature extraction and matching. PoseNet [6] is an impressive CNN-based approach for finding the pose of real images. A dataset of images was used for training Google LeNet [24]; the 6-DoF pose of the camera was used as ground truth. An improvement to PoseNet, adding an LSTM to reduce the dimensionality of the feature vector, was suggested by Ref. [7]. Meklhov et al. [8] used ResNet34 [25], which uses encoder-decoder structure to improve model accuracy. Applying an uncertainty framework to the CNN pose regressor [26] was used to improve the work in Ref. [6]. In other work, the effects of various loss functions on PoseNet's results was studied [27].

In our study we assume a $3 \mathrm{D}$ model of a city is given. Our setup is very challenging since the model and the images consist of only the coarse 3D structure of the scene without texture for computing image features. On the other hand, our images are noisefree and there are no object-level occlusions such as trees, cars, and people. In addition, we were not limited by data size, as we projected as many images as we chose. Most importantly, our goal differs from that of the aforementioned methods: whereas they focus on obtaining a better and faster solution for geo-localization, we focus on trying to understand the role of geometry in geo-localization, by systematically training and testing the same neural network on controlled datasets. 


\section{Data}

"Your network is as good as your data" is a common phrase in the world of neural networks. Our case is no different. In this respect, using a $3 \mathrm{D}$ model as the data source is highly advantageous. Using a 3D model as the data source we can sample as many images as necessary in any position, orientation, and resolution. In our study we used a simplified 3D model of Berlin [28]. The model contains only the geometry of building walls and rooftops, and it does not contain texture or fine geometric details such as window frames or doors (see Fig. 2). We consider three types of images projected from this model, based on edges, faces, and depth maps (see Fig. 1). We call them lean images since they contain no texture or structural details. The face images contain the buildings' planar facades.

Each image is defined by its camera pose: $(x, y, \theta, \phi)$, where $(x, y)$ give the position on the ground plane and $(\theta, \phi)$ are the camera yaw and pitch angles. We assume for simplicity that the picture is taken at a fixed height, and that the roll angle is fixed so that the $y$-axis is vertical. We generated several image datasets that are sampled uniformly along this $4 \mathrm{D}$ grid. The density in the $(x, y)$ domain varies between the data-sets but is fixed in the $(\theta, \phi)$ domain. A $6 \mathrm{D}$ pose vector (instead of $4 \mathrm{D}$ ) in the form of $\left(x, y, q_{1}, q_{2}, q_{3}, q_{4}\right)$, is used to represent the camera pose; $q_{1}, \ldots, q_{4}$ are the quaternion representation of the $(\theta, \phi)$ Euler angles. Each set of images was created in a predefined area of the city. The number of images in the set is determined by the size of the area and the grid sampling density. All three types of lean images were generated for each sample pose.

When dealing with lean images, care must be taken not to include empty images (for example when the camera is facing a building wall from a short distance). Such images contain almost no visual information and do not contribute to the learning process. We discard any image that has fewer than eight edges, or an image that does not contain a skyline (at least 50\% of its top-most pixel row is sky). Moreover, images of positions inside a building are irrelevant to geolocalization. We refer to such images as invalid and discard them from training and testing. See Fig. 4 for the set of invalid images in one of the considered regions.

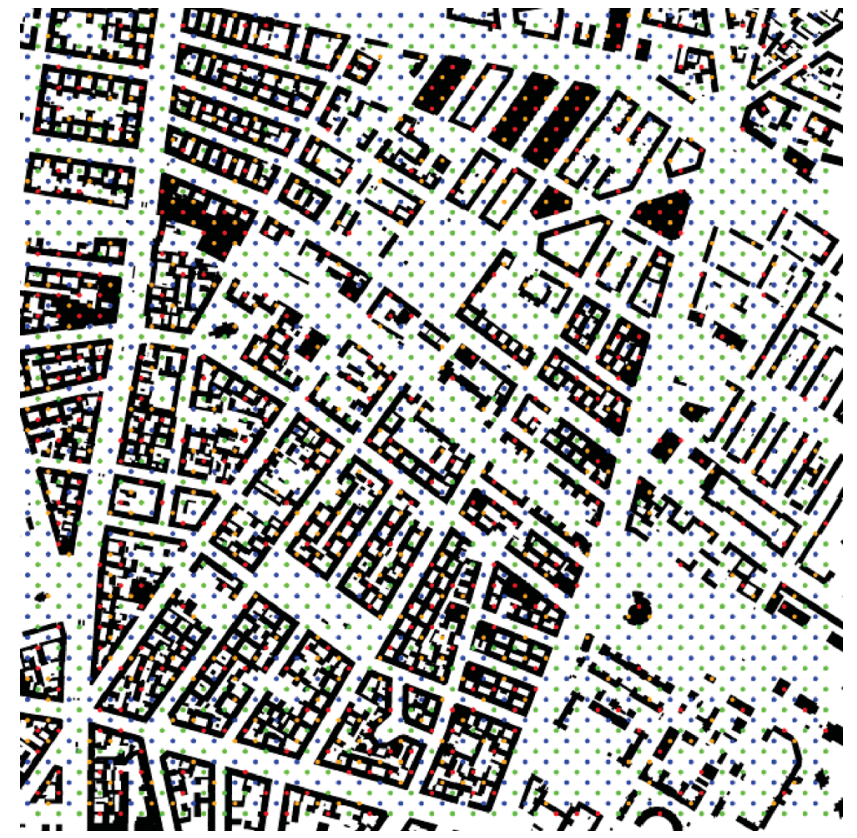

Fig. 4 Sampling positions on an area of the map. For the training set, green indicates valid samples and red invalid samples. For the test set, blue indicates valid samples and orange invalid samples.

\section{Tasks \& hypothesis}

The geo-localization task we consider is to recover the camera pose of an unseen image (not in the training set). We also consider the memorization task of retrieving the camera pose of an image from the training set. For both tasks we consider lean images as input to the network, and test several configurations of regression and classification networks (see Section 5). Our goal is to answer the following questions: (i) can a CNN be trained to perform these tasks from lean images? (ii) does geometry play a role when training the $\mathrm{CNN}$ for geo-localization?

\subsection{Memorization task}

The memorization task can be defined as a classification task where each $(x, y, \theta, \phi)$ is considered as a class. We examined whether a CNN can solve the memorization task using lean images. Given an image from the training set, we tested whether the correct camera pose could be determined. In a sense, the network is trained to overfit, and actually will not generalize. On the other hand, this would mean that the network managed to encode a very large set (hundreds of thousands) of images in some feature space as well as to efficiently match features between images to find the right pose.

We considered two specific cases. 


\subsubsection{Geometrically correlated}

In the geometrically correlated test, the camera pose for generating the image was used as ground truth for training. Hence, when the pose of nearby images is correlated, the network has access to this geometric information. We refer to this as task (A).

\subsubsection{Geometrically decorrelated}

We refer to this as task (B). We aimed here to test whether the network used the correlation between pose and image in order to learn the training data. To do so, we randomly shuffled the pose information between images so that poses were not spatially correlated with respect to the images. If no geometry is used by the CNN, the results on this training data are expected to be similar to those obtained with the real pose as ground truth. A previous study of random labels for object classification task [29] also considered randomized labels for classification, and showed that random labels only affect the time to converge.

\subsubsection{Evaluation}

A classification network may be evaluated simply by the number of correct classifications. For a regression network, the computed pose does not necessarily exactly match the pose of an image from the training set. We used the nearest neighbor (nn) grid sample to the computed pose as the pose retrieved (see Fig. 5(a)). We report the percentage of images whose correct pose is the nearest neighbor ( $1 \mathrm{nn})$ and also report the percentage of images whose correct pose is among the three nearest neighbors $(3 \mathrm{nn})$ of the computed pose. These evaluations were used for both of the memorization tasks. An additional advantage of using this measure is that it is given in grid steps and not in meter or angle units, circumventing the difficulty of comparing distances and angles, and enabling comparison of results with different grid densities (we do provide numerical $\ell_{2}$ errors in Table 2).

\subsection{Geo-localization task}

We tested whether the network can generalize and estimate the pose of an image that is not in the training set. To avoid over-fitting and allow generalization, the network was trained until the best result was achieved on a validation set. We do not expect the network to return any correct position that is outside the learned area. For this reason our test set comprises images sampled at midpoints of the training grid. These are images that are farthest from the training set samples. We refer to this as task $(\mathrm{C})$.

\subsubsection{Evaluation}

In this task we only use a regression network. A computed pose is considered correct if it lies within the same grid cell (hyper-cube) as the test sample. We report the number of correctly computed poses $(D<1)$. In addition, we considered the Manhattan distance between the hyper-cubes of the computed pose and the test sample (see Fig. 5(b)). We report the number of images for which this distance is smaller than $3(D<3)$. Note that these measures are also invariant with respect to sampling step size. Thus, we are able to compare results of experiments using sampling with different step sizes. For completeness, we also provide standard $\ell_{2}$ errors in Table 2 .

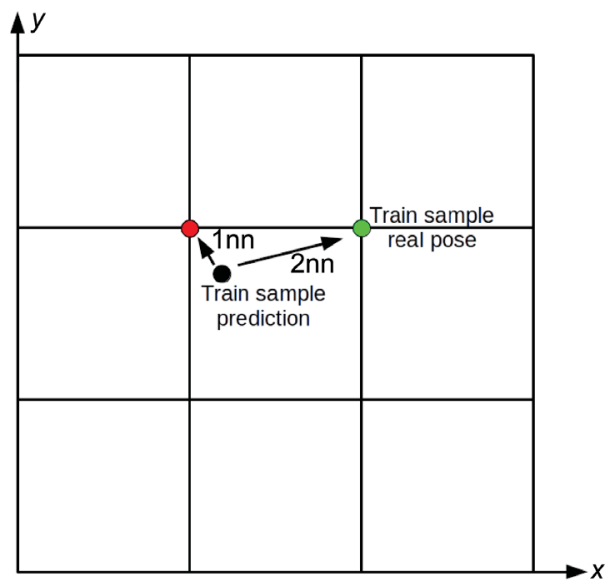

(a) Nearest neighbor

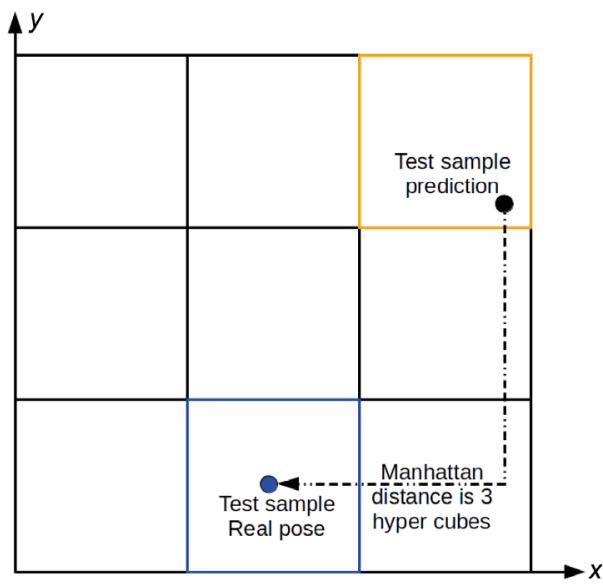

(b) Manhattan distance

Fig. 5 S2D illustration of the evaluation measures for geo-matching (a) and geo-interpolation (b). The real measures are 4D in nature. 


\section{Network}

For the geo-localization task, a regression network is more natural than a classification network. It allows the network to exploit the geometric structure and information, and to use the same trained CNN for the geo-localization task. It directly returns the pose of unseen images. Because the CNNs considered were designed for classification tasks, we follow Ref. [6] and modify the network to solve a regression task by simply removing the last softmax layer and replacing it by a fully connected layer of our result vector $\left(x, y, q_{1}, q_{2}, q_{3}, q_{4}\right)$.

Our loss function $l$ consists of a position component, $l_{\mathrm{p}}$, and an orientation component, $l_{\mathrm{o}}$. The $\ell_{2}$ distance is used for computing $l_{\mathrm{p}}$ of the position $(x, y)$, and for computing $l_{\mathrm{o}}$ of the orientation $\left(q_{1}, q_{2}, q_{3}, q_{4}\right)$. Although determining position and orientation are considered as different tasks that should have some weighting factor during the learning process, we noticed that normalizing $(x, y)$ with respect to the total area eliminates the need for such weighting. Hence, our loss function is given by the simple sum $l=l_{\mathrm{p}}+l_{\mathrm{o}}$.

We examined several CNN architectures already proved to be successful on object recognition tasks. Specifically, we tested VGG, Google LeNet [24], and ResNet50 [25], built for the ImageNet Large Scale Visual Recognition Challenge (ILSVRC) [30, 31]. In a set of preliminary experiments we found that ResNet50 combined the smallest network size in terms of parameters with the best training and testing results. Therefore, we report experiments using only the ResNet50 architecture. We trained the CNNs from scratch rather than using the pre-trained weights, since the networks we tested were trained with ImageNet, which contains real photographs. We assume that the pre-trained models are tuned for textured information that is not available in lean images. Transfer learning improves the learning rate, when moving from one AOI to another.

\section{$6 \quad$ Experiments \& results}

We tested and evaluated the regression ResNet50 network for the three tasks described in Section 4. The datasets, which are described in Section 3, are defined by the following parameters. i. Area of interest $(\mathrm{AOI}):(x, y, w, h)$ where $w$ is its width and $h$ is its height.

ii. Grid-step, $\delta$ : the distance between adjacent $(x, y)$ positions of the sampling grid: adjacent to $(x, y, \theta, \phi)$ are $(x \pm \delta, y, \theta, \phi)$ and $(x, y \pm \delta, \theta, \phi)$. The grid density in the $(\theta, \phi)$ domain was fixed.

iii. Input type: edges, faces, depth, edges + faces, edges + faces + depth. For the last two input types the images were fed to the network by stacking them channel-wise.

iv. Validation set created by randomly choosing $10 \%$ of the training samples.

v. Test set of images sampled at midpoints of the training grid.

We used various step sizes for the camera position on the grid: $\delta=10,20,40$ in model units ( 1 unit $\approx$ $1 \mathrm{~m}$ ). $\theta$ (yaw) was sampled at $5^{\circ}$ steps between $0^{\circ}$ and $360^{\circ}$, and $\phi$ (pitch) was sampled at $3^{\circ}$ steps between $0^{\circ}$ and $15^{\circ}$. The height was set to a fixed value of $z \approx 1.7 \mathrm{~m}$ above ground.

The leftmost column in Tables 1-4 indicates the amount of data used in the specified experiment (ranging from 2.5k images to 666k images). All experiments were trained for the same amount of relative time: 240 epochs in the original sense, so each network saw all the data 240 times. Training data was shuffled after each epoch.

The main results for the regression CNN are summarized in Table 1. Results are given for tasks (A-C). Three groups of rows each correspond to a different dataset, with different area and $\delta$. For each group we considered the different types of lean images and evaluated the three tasks using them as described in Section 4. Each entry is an average of three different AoIs. For completeness, Table 2 shows an example of the mean and median $\ell_{2}$ errors in pose estimation for an edges+faces experiment. Similar results were obtained in other experiments. Tables 3 and 4 show the results of testing the limitations of the CNN with respect to the sparsity of the grid $(\delta=40)$ and the size of the datasets ( $>630 \mathrm{k}$ images). We next discuss the obtained results.

\subsection{Memorization}

Very poor results were obtained for the memorization task when arbitrary poses were used as ground truth (Table 1, task (B)), i.e., when no geometric correlation between the images and their ground truth was available. The highest percentage of correct 
Table 1 Experimental results. We give the proportion of images for which a correct estimation was obtained, relative to the total number of valid images evaluated (higher is better). For geo-matching we use the nearest neighbor measure (nn), and for geo-interpolation, the Manhattan distance $(D)$

\begin{tabular}{|c|c|c|c|c|c|c|c|c|}
\hline & \multirow{4}{*}{ Input type } & \multicolumn{3}{|c|}{ Geo-matching } & \multirow{2}{*}{\multicolumn{4}{|c|}{$\begin{array}{l}\text { Geo-interpolation } \\
\text { (C) Correct pose }\end{array}$}} \\
\hline & & \multirow{3}{*}{$\begin{array}{c}\text { (B) Arbitrary pose } \\
\operatorname{1nn}\end{array}$} & \multicolumn{2}{|c|}{ (A) Correct pose } & & & & \\
\hline & & & \multirow{2}{*}{$1 \mathrm{nn}$} & \multirow{2}{*}{$3 \mathrm{nn}$} & \multicolumn{2}{|c|}{$2 \mathrm{D}(x, y)$} & \multicolumn{2}{|c|}{$4 \mathrm{D}(x, y, \theta, \phi)$} \\
\hline & & & & & $D<1$ & $D<3$ & $D<1$ & $D<3$ \\
\hline \multirow{5}{*}{$\begin{array}{l}\text { Area } 400 \times 400 \\
\text { step } 20 \\
37 \mathrm{k} \text { images }\end{array}$} & Edges & 0.45 & 0.97 & 0.99 & 0.64 & 0.82 & 0.58 & 0.75 \\
\hline & Faces & 0.35 & 0.99 & 0.99 & 0.56 & 0.76 & 0.51 & 0.69 \\
\hline & Depth & 0.23 & 0.99 & 0.99 & 0.61 & 0.79 & 0.55 & 0.72 \\
\hline & Edges+Faces & 0.29 & 0.98 & 0.99 & 0.72 & 0.88 & 0.65 & 0.82 \\
\hline & Edges+Faces+Depth & 0.24 & 0.98 & 0.99 & 0.71 & 0.88 & 0.64 & 0.81 \\
\hline \multirow{5}{*}{$\begin{array}{l}\text { Area } 400 \times 400 \\
\text { step } 10 \\
\text { 140k images }\end{array}$} & Edges & 0.11 & 0.98 & 0.98 & 0.85 & 0.94 & 0.84 & 0.93 \\
\hline & Faces & 0.05 & 0.97 & 0.97 & 0.80 & 0.90 & 0.79 & 0.88 \\
\hline & Depth & 0.06 & 0.97 & 0.97 & 0.83 & 0.92 & 0.82 & 0.91 \\
\hline & Edges+Faces & 0.09 & 0.97 & 0.97 & 0.88 & 0.96 & 0.87 & 0.95 \\
\hline & Edges+Faces+Depth & 0.08 & 0.94 & 0.95 & 0.88 & 0.96 & 0.87 & 0.95 \\
\hline \multirow{5}{*}{$\begin{array}{l}\text { Area } 800 \times 800 \\
\text { step } 20 \\
\text { 170k images }\end{array}$} & Edges & 0.06 & 0.96 & 0.96 & 0.62 & 0.78 & 0.59 & 0.75 \\
\hline & Faces & 0.01 & 0.96 & 0.96 & 0.51 & 0.68 & 0.48 & 0.65 \\
\hline & Depth & 0.01 & 0.96 & 0.97 & 0.61 & 0.77 & 0.59 & 0.73 \\
\hline & Edges+Faces & 0.04 & 0.92 & 0.93 & 0.70 & 0.86 & 0.67 & 0.83 \\
\hline & Edges+Faces+Depth & 0.03 & 0.95 & 0.96 & 0.70 & 0.85 & 0.67 & 0.81 \\
\hline
\end{tabular}

Table 2 Examples of $\ell_{2}$ errors for an experiment with Edges+Faces image type in each sub-space; spatial $(x, y)$ errors in meters, and orientation $(\theta, \phi))$ errors in degrees. As in this example, usually the errors show a long-tail distribution: many images have small errors and a few have very large errors

\begin{tabular}{|c|c|c|c|c|c|c|c|c|}
\hline & \multicolumn{4}{|c|}{ (A) Geo-matching } & \multicolumn{4}{|c|}{ (C) Geo-interpolation } \\
\hline & \multicolumn{2}{|c|}{$(x, y)$} & \multicolumn{2}{|c|}{$(\theta, \phi)$} & \multicolumn{2}{|c|}{$(x, y)$} & \multicolumn{2}{|c|}{$(\theta, \phi)$} \\
\hline & mean & median & mean & median & mean & median & mean & median \\
\hline $\begin{array}{c}\text { Area } 400 \times 400 \\
\text { step } 20 \\
37 \mathrm{k} \text { images }\end{array}$ & 3.65 & 3.26 & 0.84 & 0.69 & 26.30 & 11.26 & 10.95 & 1.84 \\
\hline $\begin{array}{c}\text { Area } 400 \times 400 \\
\text { step } 10 \\
140 \mathrm{k} \text { images }\end{array}$ & 2.37 & 2.10 & 0.57 & 0.48 & 7.99 & 3.67 & 2.65 & 0.67 \\
\hline $\begin{array}{c}\text { Area } 800 \times 800 \\
\text { step } 20 \\
170 \mathrm{k} \text { images }\end{array}$ & 5.43 & 4.71 & 0.67 & 0.54 & 40.23 & 12.28 & 9.80 & 1.40 \\
\hline
\end{tabular}

matches (45\%) was obtained for the smallest set of considered images (37k images). For the largest set (170k images), the percentage of correct matches was less than $10 \%$. As can be seen, the quality of the results decreases as the number training samples increases. In contrast, when the correct poses were used as ground truth (Table 1, task (A)), the CNN succeeded in $1 \mathrm{nn}$ localization for more than $92 \%$ of the training samples in all cases. We believe the significant differences between the two geo-matching tasks (A) and (B) is due to the network exploiting the geometric correlations when learning a metric between images. This is true, even when we consider only the memorization task, where we evaluate the over fitting of the network.
We also considered much larger datasets with more than $600 \mathrm{k}$ images (Table 3 ). The percentage of correct matches dropped to $82 \%$ for a dense grid, $\delta=10$, and to $56 \%$ for a sparser grid, $\delta=20$. For $\delta=20$ and $>600 \mathrm{k}$ images, the network capacity is probably saturated. A comparison of these results to those reported in Table 1, task (A) for the same $\delta$ values, indicates that both the number of images and the grid size determine how successfully the CNN models the data.

In addition, we tested datasets with sparser sampling in the position domain (Table 4, top 2 blocks), and in both the position and the orientation domains (Table 4, bottom 2 blocks). For sparse sampling only in the position domain, the percentage 
Table 3 Testing network learning capacity: results from a single experiment with edge image input. The network's ability to learn drops when the number of images grows beyond a certain point

\begin{tabular}{|c|c|c|c|c|c|c|}
\hline & \multicolumn{2}{|c|}{$\begin{array}{c}\text { (A) } \\
\text { Geo-matching }\end{array}$} & \multicolumn{4}{|c|}{$\begin{array}{c}(\mathrm{C}) \\
\text { Geo-interpolation }\end{array}$} \\
\hline & \multirow{2}{*}{$1 \mathrm{nn}$} & \multirow{2}{*}{$3 n n$} & \multicolumn{2}{|c|}{$2 \mathrm{D}(x, y)$} & \multicolumn{2}{|c|}{$4 \mathrm{D}(x, y, \theta, \phi)$} \\
\hline & & & $D<1$ & $D<3$ & $D<1$ & $D<3$ \\
\hline $\begin{array}{c}\text { Area } 800 \times 800 \\
\text { step } 10 \\
636 \mathrm{k} \text { images }\end{array}$ & 0.82 & 0.82 & 0.80 & 0.92 & 0.79 & 0.92 \\
\hline $\begin{array}{c}\text { Area } 1600 \times 1600 \\
\text { step } 20 \\
666 \mathrm{k} \text { images }\end{array}$ & 0.58 & 0.59 & 0.46 & 0.69 & 0.44 & 0.67 \\
\hline
\end{tabular}

of correct matches is reduced marginally. However, when reducing the sampling also in the orientation domain, the percentage of correct matches drops dramatically. This indicates that it is easier for the CNN to model a denser grid (probably because of higher geometric correlation between images), and it is easier to model fewer images (probably because of network capacity).

\subsection{Geo-localization}

Here we tested whether pose estimation by the CNN generalizes to unseen images. We used the same training as in the memorization task with the correct pose as ground truth, and tested it on images sampled from the mid point of each grid cell. We report our results with respect to $2 \mathrm{D}$ position as well as with respect to the $4 \mathrm{D}$ pose parameters (see Table 1 ).

The network was able to generalize image position with good accuracy where $\sim 70 \%$ of images are correctly positioned in their grid cell, and above
$80 \%$ of the computed poses are within three cells of the correct one. As expected, this task achieves better results on a tighter grid $(\delta=10, \sim 88 \%)$ than on a sparse grid $(\delta=20, \sim 70 \%)$. The $4 \mathrm{D}$ position error is bounded below by the $2 \mathrm{D}$ position error, and hence is greater. Moreover, the sampling rate in the orientation domain is much higher that in the location domain. Hence a small error in orientation estimate has a greater effect on the $4 \mathrm{D}$ errors. Still, the accuracy in $4 \mathrm{D}$ for $\delta=10$ is $\sim 87 \%$.

It is clear from Table 1 , task (C) that for $\delta=10$ the results are better than for $\delta=20$, even if the number of images is larger. We further explore the effect of the grid density for a sparser grid, $\delta=40$, where the percentage of correct estimates dropped significantly below $50 \%$ and $30 \%$ for $61 \mathrm{k}$ and $174 \mathrm{k}$ images, respectively (Table 4 , task (C)). For $\delta=10$ for $636 \mathrm{k}$ images, $80 \%$ of the estimates were correct (Table 3, task (C)). For this task, sparser sampling is more critical than for the memorization task as can be seen in Table 4. For very sparse sampling of the $4 \mathrm{D}$ space the network cannot really generalize to positions not seen before. Here again we believe that not only the number of images plays a role but also their density. The denser the grid, the higher the correlation between images, and hence better generalization can be obtained.

A nice application of our results is the ability to rate the distinctiveness of positions in the city. In Fig. 3 we illustrate how certain places can be easily recognized (high geo-interpolation success rate) while others are more difficult. Note, for instance,

Table 4 Low grid density results. Datasets (single experiment each) with sparse spatial sampling (top two blocks), and sparse spatial and orientation sampling (bottom two blocks) where pitch $=6^{\circ}, 7^{\circ}, \cdots, 12^{\circ}$, and yaw $=0^{\circ}, 45^{\circ}, \cdots, 315^{\circ}$. The sparser the data, the worse the results. Geo-interpolation failed for very sparse and very small datasets

\begin{tabular}{|c|c|c|c|c|c|c|c|}
\hline & \multirow{3}{*}{ Input type } & \multicolumn{2}{|c|}{ (A) Geo-matching } & \multicolumn{4}{|c|}{ (C) Geo-interpolation } \\
\hline & & \multirow{2}{*}{1 nn } & \multirow{2}{*}{$3 \mathrm{nn}$} & \multicolumn{2}{|c|}{$2 \mathrm{D}(x, y)$} & \multicolumn{2}{|c|}{$4 \mathrm{D}(x, y, \theta, \phi)$} \\
\hline & & & & $D<1$ & $D<3$ & $D<1$ & $D<3$ \\
\hline Area $800 \times 800$ & Edges & 0.90 & 0.96 & 0.39 & 0.62 & 0.30 & 0.49 \\
\hline step 40 & Edges +Faces & 0.91 & 0.97 & 0.48 & 0.72 & 0.38 & 0.59 \\
\hline $61 \mathrm{k}$ images & Edges+Faces+Depth & 0.96 & 0.98 & 0.48 & 0.72 & 0.38 & 0.58 \\
\hline Area $1600 \times 1600$ & Edges & 0.89 & 0.90 & 0.22 & 0.39 & 0.19 & 0.32 \\
\hline step 40 & Edges + Faces & 0.94 & 0.95 & 0.30 & 0.50 & 0.24 & 0.41 \\
\hline $174 \mathrm{k}$ images & Edges + Faces + Depth & 0.94 & 0.96 & 0.32 & 0.51 & 0.26 & 0.43 \\
\hline Area $800 \times 800$ & Edges & 0.40 & 0.41 & \multirow{3}{*}{\multicolumn{4}{|c|}{ Failed }} \\
\hline step 40 / sparse angles & Edges +Faces & 0.37 & 0.38 & & & & \\
\hline $2.5 \mathrm{k}$ images & Edges+Faces+Depth & 0.26 & 0.27 & & & & \\
\hline Area $1600 \times 1600$ & Edges & 0.16 & 0.18 & \multirow{3}{*}{\multicolumn{4}{|c|}{ Failed }} \\
\hline step 40 / sparse angles & Edges + Faces & 0.17 & 0.19 & & & & \\
\hline $7 \mathrm{k}$ images & Edges+Faces+Depth & 0.13 & 0.14 & & & & \\
\hline
\end{tabular}


how open spaces are more distinctive than narrow streets.

\subsection{Effect of data type}

Faces alone provide the least geometric information, and indeed in most cases were inferior to edges or depth results. Surprisingly, edges alone provide better information than depth alone for all tasks. Similar results were obtained for all data types for the memorization task for $\delta \leqslant 20$ (Table 1 ), but for a very sparse grid $\delta=40$ (Table 4 ), richer geometric information improves the results. We believe it is because the results on $\delta \leqslant 20$ were very high to begin with, using only edges.

For the geo-localization task (C), adding face information significantly improved the results, as expected. Surprisingly, depth information did not provide any significant performance gain when $\delta \leqslant 20$. This may indicate that edges+faces provide sufficient information for these cases. However, for a very sparse grid, $\delta=40$, with a relatively small number of images, adding depth information slightly improves the results (Table 4, 174k images).

For data with geometrically decorrelated pose (Task B) and for very sparse sampling (Table 4, bottom 2 blocks), the more information we added, the worse results were. The reason for this is still unclear to us. A possible explanation is that as the problem becomes more of a memorization task, the increasing information makes it harder for the CNN to find discriminant features.

\subsection{Classification}

For completeness, we also considered brute-force classifier networks. Using the number of images in the training set as output $(\approx 100 \mathrm{k}$ images $)$ creates a huge FC last layer, which results in a much larger number of parameters in comparison to the regression network. Hence we tested a network with a binary representation of 100k indexes (17 output parameters). Experiments showed that the classification CNN is able to memorize the training set extremely well, in agreement with Ref. [29]. In all experiments, using both step 10 and 20, the result exceeded $97 \%$ accuracy. On the other hand, as expected, this network gave very poor results for the geo-localization task even for $\delta=10$ (below $15 \%$ and $21 \%$ on $D<1$ and $D<3$, respectively) compared to the regression network (above 85\%). The test sample location estimation is done by feeding the CNN with a test image and than taking the location of the training sample that matches the model output. We believe this proves that such classification supervision is inadequate for the geo-localization task: the network has no incentive to create any meaningful features that could later be applied to new similar images.

\section{Conclusions}

In this work we showed that (i) CNN can achieve good results in geo-localization tasks using only lean images derived from a very simple 3D model, and (ii) geometry plays an important role in geo-localization, by achieving good results while ignoring texture and scene details. The results indicate that noise-free lean images are sufficient for solving the memorization task using a CNN, and that the use of uncorrelated images makes it nearly impossible. In addition, our results indicate that (iii) the generalization task can also be solved by CNNs when using lean images.

From a more practical perspective, it is of interest to explore whether geometric information can be used for real life geo-localization tasks, also because 3D models, e.g., from the Open Street Map project [32] are readily available.

\section{References}

[1] Se, S.; Lowe, D.; Little, J. Mobile robot localization and mapping with uncertainty using scale-invariant visual landmarks. The International Journal of Robotics Research Vol. 21, No. 8, 735-758, 2002.

[2] Lowe, D. G. Distinctive image features from scaleinvariant keypoints. International Journal of Computer Vision Vol. 60, No. 2, 91-110, 2004.

[3] Li, Y. P.; Snavely, N.; Huttenlocher, D. P. Location recognition using prioritized feature matching. In: Computer Vision - ECCV 2010. Lecture Notes in Computer Science, Vol. 6312. Daniilidis, K.; Maragos, P.; Paragios, N. Eds. Springer Berlin Heidelberg, 791804, 2010.

[4] Ramalingam, S.; Bouaziz, S.; Sturm, P. Pose estimation using both points and lines for geo-localization. In: Proceedings of the IEEE International Conference on Robotics and Automation, 4716-4723, 2011.

[5] Bansal, M.; Daniilidis, K. Geometric urban geolocalization. In: Proceedings of the IEEE Conference on Computer Vision and Pattern Recognition, 3978-3985, 2014. 
[6] Kendall, A.; Grimes, M.; Cipolla, R. PoseNet: A convolutional network for real-time 6-DOF camera relocalization. In: Proceedings of the IEEE International Conference on Computer Vision, 29382946, 2015.

[7] Walch, F.; Hazirbas, C.; Leal-Taixé, L.; Sattler, T.; Hilsenbeck, S.; Cremers, D. Image-based localization using LSTMs for structured feature correlation. In: Proceedings of the IEEE International Conference on Computer Vision, 627-637, 2017.

[8] Melekhov, I.; Ylioinas, J.; Kannala, J.; Rahtu, E. Image-based localization using hourglass networks. arXiv preprint arXiv:1703.07971, 2017.

[9] Sattler, T.; Torii, A.; Sivic, J.; Pollefeys, M.; Taira, H.; Okutomi, M.; Pajdla, T. Are large-scale 3D models really necessary for accurate visual localization? In: Proceedings of the IEEE Conference on Computer Vision and Pattern Recognition, 6175-6184, 2017.

[10] Sivic, J.; Zisserman, A. Video Google: A text retrieval approach to object matching in videos. In: Proceedings 9th IEEE International Conference on Computer Vision, 1470-1477, 2003.

[11] Robertsone, D.; Cipolla, R. An Image-based system for urban navigation. In: Proceedings of the British Machine Conference, 84.1-84.10, 2004.

[12] Hays, J.; Efros, A. A. IM2GPS: Estimating geographic information from a single image. In: Proceedings of the IEEE Conference on Computer Vision and Pattern Recognition, Anchorage, 1-8, 2008.

[13] Bergamo, A.; Sinha, S. N.; Torresani, L. Leveraging structure from motion to learn discriminative codebooks for scalable landmark classification. In: Proceedings of the IEEE Conference on Computer Vision and Pattern Recognition, 763-770, 2013.

[14] Zhang, W.; Kosecka, J. Image based localization in urban environments. In: Proceedings of the 3rd International Symposium on 3D Data Processing, Visualization, and Transmission, 33-40, 2006.

[15] Nister, D.; Stewenius, H. Scalable recognition with a vocabulary tree. In: Proceedings of the IEEE Computer Society Conference on Computer Vision and Pattern Recognition, 2161-2168, 2006.

[16] Schindler, G.; Brown, M.; Szeliski, R. Cityscale location recognition. In: Proceedings of the IEEE Conference on Computer Vision and Pattern Recognition, 1-7, 2007.

[17] Irschara, A.; Zach, C.; Frahm, J.; Bischof, H. From structure-from-motion point clouds to fast location recognition. In: Proceedings of the IEEE Conference on Computer Vision and Pattern Recognition, 25992606, 2009.
[18] Sattler, T.; Leibe, B.; Kobbelt, L. Fast imagebased localization using direct 2D-to-3D matching. In: Proceedings of the International Conference on Computer Vision, 667-674, 2011.

[19] Matei, B. C.; Vander Valk, N.; Zhu, Z.; Cheng, H.; Sawhney, H. S. Image to LIDAR matching for geotagging in urban environments. In: Proceedings of the IEEE Workshop on Applications of Computer Vision, 413-420, 2013.

[20] Svärm, L.; Enqvist, O.; Oskarsson, M.; Kahl, F. Accurate localization and pose estimation for large 3D models. In: Proceedings of the IEEE Conference on Computer Vision and Pattern Recognition, 532-539, 2014.

[21] Baatz, G.; Saurer, O.; Köser, K.; Pollefeys, M. Large scale visual geo-localization of images in mountainous terrain. In: Computer Vision - ECCV 2012. Lecture Notes in Computer Science, Vol. 7573. Fitzgibbon, A.; Lazebnik, S.; Perona, P.; Sato, Y.; Schmid, C. Eds. Springer Berlin Heidelberg, 517-530, 2012.

[22] Svarm, L.; Enqvist, O.; Kahl, F.; Oskarsson, M. Cityscale localization for cameras with known vertical direction. IEEE Transactions on Pattern Analysis and Machine Intelligence Vol. 39, No. 7, 1455-1461, 2017.

[23] Piasco, N.; Sidibé, D.; Demonceaux, C.; Gouet-Brunet, V. A survey on visual-based localization: On the benefit of heterogeneous data. Pattern Recognition Vol. 74, 90109, 2018.

[24] Szegedy, C.; Liu, W.; Jia, Y.; Sermanet, P.; Reed, S.; Anguelov, D.; Erhan, D.; Vanhoucke, V.; Rabinovich, A. Going deeper with convolutions. In: Proceedings of the IEEE Conference on Computer Vision and Pattern Recognition, 1-9, 2015.

[25] He, K.; Zhang, X.; Ren, S.; Sun, J. Deep residual learning for image recognition. In: Proceedings of the IEEE Conference on Computer Vision and Pattern Recognition, 770-778, 2016.

[26] Kendall, A.; Cipolla, R. Modelling uncertainty in deep learning for camera relocalization. In: Proceedings of the IEEE International Conference on Robotics and Automation, 4762-4769, 2016.

[27] Kendall, A.; Cipolla, R. Geometric loss functions for camera pose regression with deep learning. In: Proceedings of the IEEE Conference on Computer Vision and Pattern Recognition, 6555-6564, 2017.

[28] Berlin Partner für Wirtschaft und Technologie GmbH. Berlin 3D city model. 2016. Available at https://www.businesslocationcenter.de/en/WA/B/ seite0.jsp.

[29] Zhang, C.; Bengio, S.; Hardt, M.; Recht, B.; Vinyals, O. Understanding deep learning requires rethinking generalization. arXiv preprint arXiv:1611.03530, 2016. 
[30] Russakovsky, O.; Deng, J.; Su, H.; Krause, J.; Satheesh, S.; Ma, S.; Huang, Z.; Karpathy, A.; Khosla, A.; Bernstein, M.; Berg, A. C.; Fei-Fei, L. ImageNet large scale visual recognition challenge. International Journal of Computer Vision Vol. 115, No. 3, 211-252, 2015.

[31] Deng, J.; Dong, W.; Socher, R.; Li, L.-J.; Li, K.; Fei-Fei, L. ImageNet: A large-scale hierarchical image database. In: Proceedings of the IEEE Conference on Computer Vision and Pattern Recognition, 248-255, 2009.

[32] OpenStreetMap Wiki contributors. OSM-3D.org. OpenStreetMap Wiki, 2018. Available at https://wiki.openstreetmap.org/w/index.php?title= OSM-3D.org\&oldid=2025859.

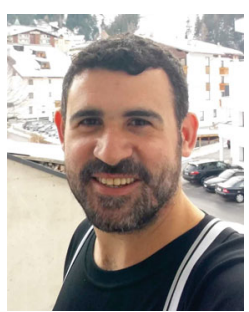

Moti Kadosh obtained his M.Sc. degree with honours from the Department of Electrical Engineering in Tel-Aviv University. He is working as a machine learning researcher in a medical AI firm specializing in interpreting medical images. His main fields of interest are computer vision, machine learning, deep learning, and computer graphics.

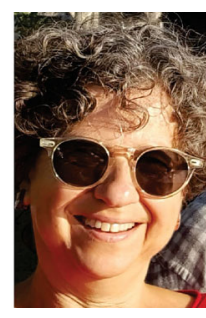

Yael Moses is a professor in the Efi Arazi School of Computer Science at the Interdisciplinary Center, Herzliya, which she joined in 1999. She received her Ph.D. degree from the Weizmann Institute of Science in 1993. She was a post-doctoral fellow with the Robotics Group of Oxford University in 1993-1994, and at the Weizmann Institute in 1994-1997. Her research interests include multi-camera systems, visual surveillance, analyzing CrowdCam images, and music transcription from video.

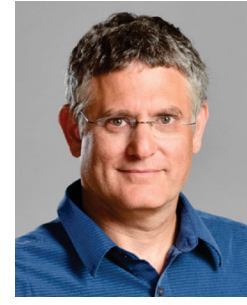

Ariel Shamir is Dean of the Efi Arazi School of Computer Science at the Interdisciplinary Center in Israel. He received his Ph.D. degree in computer science in 2000 from the Hebrew University in Jerusalem, and spent two years as a postdoc at the University of Texas in Austin. Prof. Shamir has numerous publications and a number of patents, and was named one of the most highly cited researchers on the Thomson Reuters list in 2015. He has broad commercial experience consulting for various companies including Disney Research, Mitsubishi Electric, PrimeSense (now Apple), Verisk, and more. Prof. Shamir specializes in geometric modeling, computer graphics, image processing, and machine learning. He is a member of ACM SIGGRAPH, IEEE Computer, AsiaGraphics, and EuroGraphics associations.

Open Access This article is licensed under a Creative Commons Attribution 4.0 International License, which permits use, sharing, adaptation, distribution and reproduction in any medium or format, as long as you give appropriate credit to the original author(s) and the source, provide a link to the Creative Commons licence, and indicate if changes were made.

The images or other third party material in this article are included in the article's Creative Commons licence, unless indicated otherwise in a credit line to the material. If material is not included in the article's Creative Commons licence and your intended use is not permitted by statutory regulation or exceeds the permitted use, you will need to obtain permission directly from the copyright holder.

To view a copy of this licence, visit http:// creativecommons.org/licenses/by/4.0/.

Other papers from this open access journal are available free of charge from http://www.springer.com/journal/41095. To submit a manuscript, please go to https://www. editorialmanager.com/cvmj. 